\title{
Vortices in a mesoscopic superconducting circular sector
}

\author{
Edson Sardella, Paulo Noronha Lisboa-Filho, and André Luiz Malvezzi \\ Departamento de Física, Faculdade de Ciências, Universidade Estadual Paulista-UNESP, Caixa Postal 473, 17033-360, \\ Bauru-São Paulo, Brazil
}

(Received 12 August 2007; revised manuscript received 29 January 2008; published 6 March 2008)

\begin{abstract}
In the present paper we develop an algorithm to solve the time dependent Ginzburg-Landau equations, by using the link variables technique, for circular geometries. In addition, we evaluate the Helmholtz and Gibbs free energy, the magnetization, and the number of vortices. This algorithm is applied to a circular sector. We evaluate the superconduting-normal magnetic field transition, the magnetization, and the superconducting density. Our results point out that, as we reduce the superconducting area, the nucleation field increases. Nevertheless, as the angular width of the circular sector goes to small values the asymptotic behavior is independent of the sample area. We also show that the value of the first nucleation field is approximately the same independently of the form of the circular sector. Furthermore, we study the nucleation of giant and multivortex states for the different shapes of the present geometry.
\end{abstract}

DOI: $10.1103 /$ PhysRevB.77.104508

PACS number(s): 74.25.-q, 74.20.De, 74.78.Na

\section{INTRODUCTION}

The advances in the technologies of nanofabrication in the last few decades allowed intensive investigation efforts in nanostructured superconductors, both in the experimental and theoretical fronts. ${ }^{1,2}$ It is well-known that, for very confined geometries, the superconducting-normal ( $\mathrm{SN}$ ) magnetic field transition is increased extraordinarily. It was experimentally observed that for an $\mathrm{Al}$ square of very thin film with a size of a few micrometers the upper critical field $H_{c 2}(T)$ can be increased up to 3.32 with the inclusion of defects, 2.01 larger than the usual value of $H_{c 2}(T) .{ }^{2}$ Furthermore, numerical simulations carried out in a circular wedge (see Ref. 3 and references therein) have shown that by keeping the area of this geometry constant, the SN transition field is a uniform increasing function with decreasing angular width $\Theta$ and diverges as the angle goes to zero.

Another important issue in confined geometries is the occurrence of giant vortices. The experimental observation of a giant vortex in a mesoscopic superconductor is still a controversial issue. Through multi-small-tunnel-junction measurements in an $\mathrm{Al}$ thin disk film, Kanda et al. ${ }^{4}$ have argued that, as the vorticity increases, giant vortex configuration will occur. On the other hand, scanning superconducting quantum interference device (SQUID) microscopy on $\mathrm{Nb}$ thin film, both square and triangle, cannot guarantee giant vortex configurations, at least for low vorticity. ${ }^{5,6}$ In other words, the authors of these references do not have sufficient resolution in some pictures to affirm that giant configurations are present. Despite this minor difference in experimental observations, most of the works indicate the occurrence of giant vortices in confined geometries. A recent work on a Bitter pattern decoration experiment strongly suggests the formation of giant vortices in small superconducting disks. ${ }^{7}$

Early numerical simulations of the present authors ${ }^{8}$ have shown the dynamic of the nucleation of a giant and multivortex state before they set into an equilibrium configuration for a square geometry. The results of these simulations also reenforce the existence of giant vortex states as well as the time dependence of the nucleation of multi- and giant vortex systems.
It is well-known that the phenomenology of superconductivity can be described by the time dependent GinzburgLandau (TDGL) equations. ${ }^{10}$ The present contribution uses the TDGL approach to address the issues above, namely, of the nucleation of vortices in confined geometries and the behavior of the transition field for a deformable geometry. For this, we have chosen a circular sector (see Fig. 1), where we can arbitrarily change its shape. To our best knowledge, the discretization of the TDGL equations, by using the link variables technique, has been done only in rectangular coordinates. ${ }^{11,12}$ So, we will extend this algorithm to circular geometries by using polar coordinates. Our procedure makes it possible to generalize the algorithm to any geometry. The key point in such a problem is how to write the auxiliary fields appropriately according to the system of coordinates, making the development of the present algorithm a specific algorithm necessary. Otherwise, the purpose of generaliza-

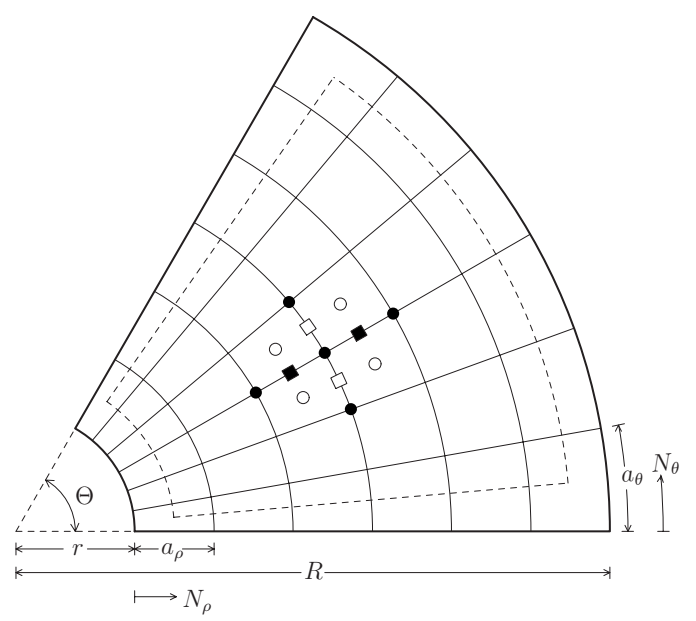

FIG. 1. The computational mesh in polar coordinates used for the evaluation of $\psi_{i, j}(\bullet$, vertex point $) ; h_{z, i, j}$ and $L_{i, j}(\bigcirc$, cell point $)$; $A_{\rho, i, j}$ and $U_{\rho, i, j}\left(\boldsymbol{\square}\right.$, link point); and $A_{\theta, i, j}$ and $U_{\theta, i, j}$ ( $\square$, link point). The superconducting domain is delimited by the dashed line $\partial \Omega_{\mathrm{SC}}$, and superconducting layer is surrounded by the solid line $\partial \Omega$. Other details of the figure are described in Sec. III. 
tion will not be achieved. We anticipate that the two main points of the present work will show that: (a) as we decrease the area of the circular sector, the transition field may increase for large angles, but all the curves will collapse into the asymptotic behavior $H / H_{c 2}(T)=\sqrt{3} / \Theta$; and (b) only the confinement of vortices is not sufficient to obtain a giant vortex state, but also the geometry is very important to favor the nucleation of such configurations; by confinement we mean the space available for the nucleation of vortices inside the sample. The geometry we have chosen does not allow us to assure clearly the nucleation of the giant vortex. For a circular sector of several angular widths, ranging from $45^{\circ}$ to $180^{\circ}$, having the same area as a disk, a square, and a triangle, we did not observe the occurrence of a giant vortex as previous numerical simulations have predicted for those geometries. ${ }^{9}$ In addition, we will show that the criterion used for nucleation of a giant vortex may lead us to nonconclusive pictures, at least for the geometry under the present investigation.

The paper is outlined as follows. In Sec. II we write the TDGL equations in a gauge invariant form by using the auxiliary field in polar coordinates. In Sec. III we develop the algorithm we use to solve the TDGL equations: we define the mesh used to discretize the TDGL equations for a circular sector, the discrete variables which are evaluated in the mesh, the boundary conditions, and, finally, the important physical quantities which will be extracted from the numerical setup are determined. In Sec. IV we present and discuss the results of the numerical simulations for certain parameters of a superconducting circular sector.

\section{TDGL EQUATIONS}

The properties of the superconducting state are usually described by the complex order parameter $\psi$, for which the absolute square value $|\psi|^{2}$ represents the superfluid density, and the vector potential $\mathbf{A}$, which is related to the local magnetic field as $\mathbf{h}=\nabla \times \mathbf{A}$. If either a transport current or an external electric field is present, the scalar potential $\Phi$ must be taken into account. These quantities are determined by the TDGL equations, which in the nondimensional version are given by

$$
\begin{gathered}
\frac{\partial \psi}{\partial t}+i \Phi \psi=-\mathbf{D} \cdot \mathbf{D} \psi+(1-T) \psi\left(1-|\psi|^{2}\right), \\
\beta\left(\frac{\partial \mathbf{A}}{\partial t}+\nabla \Phi\right)=(1-T) \operatorname{Re}[\bar{\psi} \mathbf{D} \psi]-\kappa^{2} \nabla \times \mathbf{h},
\end{gathered}
$$

where $T$ is the temperature in units of the critical temperature; lengths are in units of $\xi(0)$, the coherence length is at zero temperature, and fields in units of $H_{c 2}(0)$, the upper critical field is at zero temperature; $\beta$ is the ratio between the relaxation times of the vector potential and the order parameter; $\kappa$ is the Ginzburg-Landau parameter which is material dependent; the operator $\mathbf{D}=-i \nabla-\mathbf{A}$; Re indicates the real part of a complex variable and the overbar means the complex conjugation (for more details, see Refs. 8, 11, and 12). Here, we will neglect the $z$ dependence on the order param- eter. This is valid only if the system is infinite along the $z$ direction. We could also apply the $z$ invariant approach to the very special case of a very thin film of thickness $d \ll 1$. It has been argued in Ref. 13 that, if the system is finite in the $z$ direction, the order parameter can be expanded in a Fourier series satisfying the appropriate boundary conditions. In this same reference, it is shown that the main contribution to the order parameter corresponds to the zero wave-vector term of the series, provided that $d \ll 1$. This term is just the twodimensional solution of the first Ginzburg-Landau equation, which is invariant along the $z$ direction. However, in this case, $\kappa^{2}$ is replaced by an effective Ginzburg-Landau parameter $\kappa_{e f f}^{2}=\kappa^{2} / d$ (for instance, see Refs. 14 and 15). Therefore this approximation could give us some information of the physics involved in a real system, provided that $d \ll 1$. The generalization to a system of arbitrary thickness should not present any difficulty.

It is convenient to introduce the auxiliary vector field $\mathcal{U}$ $=\left(\mathcal{U}_{\rho}, \mathcal{U}_{\theta}\right)$ in polar coordinates, which is defined by

$$
\begin{gathered}
\mathcal{U}_{\rho}(\rho, \theta)=\exp \left(-i \int_{\rho_{0}}^{\rho} A_{\rho}(\theta, \xi) d \xi\right), \\
\mathcal{U}_{\theta}(\rho, \theta)=\exp \left(-i \int_{\theta_{0}}^{\theta} A_{\theta}(\xi, \rho) \rho d \xi\right),
\end{gathered}
$$

where $\left(\rho_{0}, \theta_{0}\right)$ is an arbitrary reference point. For the sake of brevity we omit the time dependence on the fields.

Notice that

$$
\frac{\partial \mathcal{U}_{\rho}}{\partial \rho}=-i A_{\rho} \mathcal{U}_{\rho}, \quad \frac{1}{\rho} \frac{\partial \mathcal{U}_{\theta}}{\partial \theta}=-i A_{\partial} \mathcal{U}_{\theta},
$$

and that

$$
D_{\rho} \psi=-i \overline{\mathcal{U}}_{\rho} \frac{\partial\left(\mathcal{U}_{\rho} \psi\right)}{\partial \rho}, \quad D_{\theta} \psi=-i \frac{\overline{\mathcal{U}}_{\theta}}{\rho} \frac{\partial\left(\mathcal{U}_{\theta} \psi\right)}{\partial \theta} .
$$

Upon using these two last equations recursively, we obtain

$$
D_{\rho}^{2} \psi=-\overline{\mathcal{U}}_{\rho} \frac{\partial^{2}\left(\mathcal{U}_{\rho} \psi\right)}{\partial \rho^{2}}, \quad D_{\theta}^{2} \psi=-\frac{\overline{\mathcal{U}}_{\theta}}{\rho^{2}} \frac{\partial^{2}\left(\mathcal{U}_{\theta} \psi\right)}{\partial \theta^{2}} .
$$

As a consequence, we obtain for the kinetic term in the first TDGL equation

$$
\begin{aligned}
\mathbf{D} \cdot \mathbf{D} \psi= & D_{\rho}^{2} \psi+D_{\theta}^{2} \psi-\frac{i}{\rho} D_{\rho} \psi=-\frac{\overline{\mathcal{U}}_{\rho}}{\rho} \frac{\partial}{\partial \rho}\left[\rho \frac{\partial\left(\mathcal{U}_{\rho} \psi\right)}{\partial \rho}\right] \\
& -\frac{\overline{\mathcal{U}}_{\theta}}{\rho^{2}} \frac{\partial^{2}\left(\mathcal{U}_{\theta} \psi\right)}{\partial \theta^{2}} .
\end{aligned}
$$

From Eqs. (4), it can also be easily proved that 


$$
\begin{aligned}
\operatorname{Re}\left[\bar{\psi} D_{\rho} \psi\right] & =\operatorname{Im}\left[\overline{\mathcal{U}}_{\rho} \bar{\psi} \frac{\partial\left(\mathcal{U}_{\rho} \psi\right)}{\partial \rho}\right], \quad \operatorname{Re}\left[\bar{\psi} D_{\theta} \psi\right] \\
& =\operatorname{Im}\left[\frac{\overline{\mathcal{U}}_{\theta} \bar{\psi}}{\rho} \frac{\partial\left(\mathcal{U}_{\theta} \psi\right)}{\partial \theta}\right],
\end{aligned}
$$

where Im indicates the imaginary part of a complex variable.

Finally, by using Eqs. (6) and (7), the TDGL equations of Eq. (1) can be rewritten as

$$
\begin{aligned}
& \frac{\partial \psi}{\partial t}+i \Phi \psi=\frac{\overline{\mathcal{U}}_{\rho}}{\rho} \frac{\partial}{\partial \rho}\left[\rho \frac{\partial\left(\mathcal{U}_{\rho} \psi\right)}{\partial \rho}\right]+\frac{\overline{\mathcal{U}}_{\theta}}{\rho^{2}} \frac{\partial^{2}\left(\mathcal{U}_{\theta} \psi\right)}{\partial \theta^{2}}+(1-T) \psi(1 \\
& \left.-|\psi|^{2}\right) \text {, } \\
& \beta\left(\frac{\partial A_{\rho}}{\partial t}+\frac{\partial \Phi}{\partial \rho}\right)=(1-T) \operatorname{Im}\left[\overline{\mathcal{U}}_{\rho} \bar{\psi} \frac{\partial\left(\mathcal{U}_{\rho} \psi\right)}{\partial \rho}\right]-\kappa_{e f f}^{2} \frac{1}{\rho} \frac{\partial h_{z}}{\partial \theta}, \\
& \beta\left(\frac{\partial A_{\theta}}{\partial t}+\frac{1}{\rho} \frac{\partial \Phi}{\partial \theta}\right)=(1-T) \operatorname{Im}\left[\frac{\overline{\mathcal{U}}_{\theta} \bar{\psi}}{\rho} \frac{\partial\left(\mathcal{U}_{\theta} \psi\right)}{\partial \theta}\right]+\kappa_{\text {eff }}^{2} \frac{\partial h_{z}}{\partial \rho} .
\end{aligned}
$$

Disregarding the nonlinear term, the first TDGL equation written as above resembles a diffusion equation, except by the fact that the Laplacian appears with different weights. The weights depend locally on the components of the auxiliary field $\mathcal{U}$. Written like in Eq. (8), the TDGL equations are gauge invariant, that is, they do not change their form under any transformation $\psi^{\prime}=\psi e^{i \chi}, \quad \mathbf{A}^{\prime}=\mathbf{A}+\nabla \chi, \quad$ and $\quad \Phi^{\prime}=\Phi$ $-\partial \chi / \partial t$. This is a very important point for any discretization procedure of the TDGL equations. Otherwise, we may obtain nonphysical numerical solutions. In what follows, we will work in the zero-electric potential gauge $\Phi^{\prime}=0$, since in the present scenario no electrical field is considered. Other possible gauges have been discussed in detail in Ref. 16.

\section{NUMERICAL METHOD}

We will discretize the TDGL equations of Eq. (8) on a circular sector as illustrated in Fig. 1. The mesh consists of $N_{\rho} \times N_{\theta}$ cells with size $\left(a_{\rho}, a_{\theta}\right)$ in polar coordinates. The circular sector has internal radius $r$ and external $R$; $\Theta$ is its angular width. Let $\left(\rho_{i}, \theta_{j}\right)$ be a vertex point in the mesh, where $\rho_{i+1}=\rho_{i}+a_{\rho}, \quad \theta_{j+1}=\theta_{j}+a_{\theta}$ for all $\left\{1 \leqslant i \leqslant N_{\rho}, 1 \leqslant j\right.$ $\left.\leqslant N_{\theta}\right\} ; \rho_{1}=r$ and $\theta_{1}=0$; this particular choice for the initial value of the angle does not imply a loss of generality since the system is invariant under any rotation. The superconducting domain is comprehended by $\Omega_{\mathrm{SC}}=\left\{\rho_{1}+a_{\rho} / 2<\rho<\rho_{N_{\rho}}\right.$ $\left.+a_{\rho} / 2, a_{\theta} / 2<\theta<\theta_{N_{\theta}}+a_{\theta} / 2\right\}$. The superconducting region is surrounded by a thin superconducting layer of width $a_{\rho} / 2$ in the radial direction conveniently detached from the superconductor. Both regions are inside the domain $\Omega=\left\{\rho_{1}<\rho\right.$ $\left.<\rho_{N_{\rho}+1}, 0<\theta<\theta_{N_{\theta^{+}}}\right\}$. We denote by $\partial \Omega_{\mathrm{SC}}$ the interface between the superconductor and the external superconducting layer, and by $\partial \Omega$ the superconducting layer-vacuum interface. The boundary conditions will be employed at the $\partial \Omega_{\mathrm{SC}}$ interface rather than $\partial \Omega$. The real interface is a superconductor-vacuum $\partial \Omega$. We introduce a very thin superconducting layer in between the superconductor and the vacuum just as an artifact to avoid the divergence of the derivative of the order parameter at the superconductorvacuum interface, although both regions consist of the same superconduting material. This usual procedure is used in the link variable formalism, not only to avoid divergence of the derivative of the order parameter at the $\partial \Omega$ interface, but also to obtain a discretization of the Ginzburg-Landau equations which preserves the gauge invariance of these equations (see Refs. 11 and 17 for more details). The thinner the superconducting layer is, the better our approximation will be.

Let us define the following discrete variables.

(1) The vertex points

$$
\begin{gathered}
\rho_{i}=r+(i-1) a_{\rho}, \quad 1 \leqslant i \leqslant N_{\rho}+1, \\
\theta_{j}=(j-1) a_{\theta}, \quad 1 \leqslant j \leqslant N_{\theta}+1 .
\end{gathered}
$$

The points $\left(\rho_{i+1 / 2}=\rho_{i}+a_{\rho} / 2, \theta_{i+1 / 2}=\theta_{i}+a_{\theta} / 2\right)$ are called the cell points. The points $\left(\rho_{i+1 / 2}, \theta_{j}\right)$ and $\left(\rho_{i}, \theta_{j+1 / 2}\right)$ are the link points in the radial and transversal directions, respectively (see Fig. 1).

(2) The order parameter

$$
\psi_{i, j}=\psi\left(\rho_{i}, \theta_{j}\right)
$$

for all $\left\{1 \leqslant i \leqslant N_{\rho}+1,1 \leqslant j \leqslant N_{\theta}+1\right\}$.

(3) The vector potential

$$
A_{\rho, i, j}=A_{\rho}\left(\rho_{i+1 / 2}, \theta_{j}\right), \quad A_{\theta, i, j}=A_{\theta}\left(\rho_{i}, \theta_{j+1 / 2}\right),
$$

for all $\left\{1 \leqslant i \leqslant N_{\rho}, 1 \leqslant j \leqslant N_{\theta}+1\right\}$ and $\left\{1 \leqslant i \leqslant N_{\rho}+1,1 \leqslant j\right.$ $\left.\leqslant N_{\theta}\right\}$, respectively.

(4) The link variables

$$
\begin{gathered}
U_{\rho, i, j}=\overline{\mathcal{U}}_{\rho}\left(\rho_{i}, \theta_{j}\right) \mathcal{U}_{\rho}\left(\rho_{i+1}, \theta_{j}\right)=\exp \left(-i a_{\rho} A_{\rho, i, j}\right), \\
U_{\theta, i, j}=\overline{\mathcal{U}}_{\theta}\left(\rho_{i}, \theta_{j}\right) \mathcal{U}_{\theta}\left(\rho_{i}, \theta_{j+1}\right)=\exp \left(-i \rho_{i} a_{\theta} A_{\theta, i, j}\right),
\end{gathered}
$$

for all $\left\{1 \leqslant i \leqslant N_{\rho}, 1 \leqslant j \leqslant N_{\theta}+1\right\}$ and $\left\{1 \leqslant i \leqslant N_{\rho}+1,1 \leqslant j\right.$ $\left.\leqslant N_{\theta}\right\}$, respectively.

(5) The local magnetic field

$$
h_{z, i, j}=h_{z}\left(\rho_{i+1 / 2}, \theta_{j+1 / 2}\right),
$$

for all $\left\{1 \leqslant i \leqslant N_{\rho}, 1 \leqslant j \leqslant N_{\theta}\right\}$.

In what follows, it will be important to define the following discrete variable:

$$
\begin{aligned}
L_{i, j}= & \exp \left(-i \oint_{\partial \mathcal{D}} \mathbf{A} \cdot d \mathbf{r}\right)=\exp \left(-i \int_{\mathcal{D}} h_{z} \rho d \rho d \theta\right)=\exp ( \\
& \left.-i a_{\rho} \rho_{i+1 / 2} a_{\theta} h_{z, i, j}\right)
\end{aligned}
$$

for all $\left\{1 \leqslant i \leqslant N_{\rho}, 1 \leqslant j \leqslant N_{\theta}\right\}$, where $\mathcal{D}$ is the domain of a unit cell limited by a closed path $\partial \mathcal{D}$. The use of Stoke's theorem and the midpoint rule for numerical integration have been made. A simple inspection of Eq. (14) leads to

$$
L_{i, j}=U_{\rho, i, j} U_{\theta, i+1, j} \bar{U}_{\rho, i, j+1} \bar{U}_{\theta, i, j}
$$

In Appendix A, by using this approach, and on using the one-step forward-difference Euler scheme with time step $\Delta t$, 
we descretized the TDGL equations. We find the following recurrence relations for the order parameter and the link variables:

$$
\begin{gathered}
\psi_{i, j}(t+\Delta t)=\psi_{i, j}(t)+\mathcal{F}_{\psi, i, j}(t) \Delta t, \\
U_{\alpha, i, j}(t+\Delta t)=U_{\alpha, i, j}(t) \exp \left(-\frac{i}{\beta} \mathcal{F}_{U_{\alpha} i, j}(t) \Delta t\right),
\end{gathered}
$$

where the functions $\mathcal{F}_{\psi, i, j}(t), \mathcal{F}_{U_{\alpha} i, j}(t)$, with $\alpha=(\rho, \theta)$, are defined in Appendix A.

Notice that Eqs. (16) were written in such a manner they guarantee the link variables are unimodular functions. The first recurrence relation runs for all interior vertex points of $\Omega_{\mathrm{SC}}$, that is, $\left\{2 \leqslant i \leqslant N_{\rho}, 2 \leqslant j \leqslant N_{\theta}\right\}$; the second ones run for all link points in the interior of $\Omega$, that is, $\left\{1 \leqslant i \leqslant N_{\rho}, 2 \leqslant j\right.$ $\left.\leqslant N_{\theta}\right\}$ for $\alpha=\rho$, and $\left\{2 \leqslant i \leqslant N_{\rho}, 1 \leqslant j \leqslant N_{\theta}\right\}$ for $\alpha=\theta$. At the edge points of $\Omega$, the values of the discrete variables will be evaluated using the boundary conditions (see the next section).

There is a severe limitation on the choice of the time step $\Delta t$ such that the recurrence relations converge. We have noticed that the condition for stability is assured by the following practical rule:

$$
\Delta t \leqslant \min \left\{\frac{\delta^{2}}{4}, \frac{\delta^{2} \beta}{4 \kappa^{2}}\right\}, \quad \delta^{2}=\frac{2}{\frac{1}{a_{\rho}^{2}}+\frac{1}{r^{2} a_{\theta}^{2}}} .
$$

Notice that the stability is controlled by the size of the smallest unit cell. The smaller the value of $r$, the more severe the restriction on the time step becomes. Perhaps in this case, it would be more convenient to use either a semi- or a full implicit scheme to solve TDGL equations, which are usually unconditionally convergent.

Let us now discuss the boundary conditions. Let $\mathbf{n}$ be a unit vector normal to the $\partial \Omega_{\mathrm{SC}}$ interface and directed outward to the domain $\Omega_{\mathrm{SC}}$. We will assume that the normal current density vanishes at the $\partial \Omega_{S C}$ interface, that is, $\mathbf{D} \psi \cdot \mathbf{n}=0$. By using Eqs. (4), it can be shown that the discrete implementation of this condition is as follows:

$$
\begin{aligned}
& \psi_{1, j}=U_{\rho, 1, j} \psi_{2, j}, \quad \psi_{N_{\rho}+1, j}=\bar{U}_{\rho, N_{\rho}, j} \psi_{N_{\rho}, j}, \\
& \psi_{i, 1}=U_{\theta, i, 1} \psi_{i, 2}, \quad \psi_{i, N_{\theta}+1}=\bar{U}_{\theta, i, N_{\theta}} \psi_{i, N_{\theta}} .
\end{aligned}
$$

The first two equations run for all values of $\left\{2 \leqslant j \leqslant N_{\theta}\right\}$, and the second ones for all values of $\left\{2 \leqslant i \leqslant N_{\rho}\right\}$. At the corner vertex points of the domain $\Omega$ it is not necessary to run the recurrence relations (16).

These last four equations update the values of the order parameter at any vertex point at the $\partial \Omega$ interface. The values of the link variables at this interface will be updated by using the fact that the $z$ component of the magnetic field is continuous at the interface $\partial \Omega_{\mathrm{SC}}$, that is, $h_{z, 1, j}=h_{z, N_{\rho}, j}=h_{z, i, 1}$ $=h_{z, i, N_{\theta}}=H$, which is the external applied magnetic field. Consequently, from Eqs. (14) and (15), the link variables are updated according to

$$
L_{i, j}=\exp \left(-i a_{\rho} \rho_{i+1 / 2} a_{\theta} H\right),
$$

which runs for all edge points at the interface $\partial \Omega$.

In Appendix B we also present the derivation of some very important physical quantities like the free energy, magnetization, and vorticity.

\section{RESULTS AND DISCUSSION}

The recurrence relations derived in the previous section were implemented as follows. We started from the Meissner state, where $\psi=1$ and $U_{\rho}=U_{\theta}=1$ everywhere as the initial condition. Then we let the time evolve until the system achieves the stationary state. This is done by keeping the external applied magnetic field $H$ constant. Next, we ramp up the applied field by an amount of $\Delta H$. The stationary solution for $H$ is then used as the initial state to determine the solution for $H+\Delta H$, and so on. Usually we started from zero field and increased $H$ until superconductivity is destroyed. As a criterion for termination of the simulation, we monitored the Gibbs free energy as a function of $H$. When the value of this quantity changes its sign, then the transition from the superconducting to the normal state sets in.

We use the following criterion to obtain the stationary state: if the highest difference $\|\psi(t)|-| \psi(t+\Delta t)\|$, for any vertex point in the mesh, is smaller than a certain precision $\epsilon$, then we go over to the next field. We have worked with a precision of $\epsilon=10^{-6}$ for $\Theta=45^{\circ}$ and $\epsilon=10^{-5}$ for the other angular widths. The reason for taking different precisions is as follows. The initial state is taken as the stationary state from the previous value of the applied magnetic field. So, along the simulation may there be accumulation of error as the magnetic field increase. Since the SN transition field is higher for smaller angular widths, as will be seen in what follows, to overcome any divergence difficulties we set a higher precision for these cases.

The parameters used in our numerical simulations were $\kappa=0.28$, which is a typical value for thin $\mathrm{Al}$ samples, ${ }^{2} d$ $=0.1, T=0$, and $\beta=1$. The internal radius and the area of the circular sector were taken fixed for any value of angular width $\Theta$. We used $r=1 / \pi$ and $S=16 \pi$ for the area, such that the external radius is given by $R=\sqrt{32 \pi / \Theta+r^{2}}$. The reason for taking these parameters as such is because it makes possible the comparison between our results and previous ones (see Ref. 9 and references therein). The size of the mesh varied according to the value of $\Theta$. As a criterion we have taken the length of the largest unit cell no larger then 0.25 $\times 0.25$. Since the order parameter varied most significantly over a distance $\xi(T)$ (in real units), we are certain of not losing this variation within this criterion. We ramp up the applied magnetic field, typically in steps of $\Delta H=10^{-3}$.

In Fig. 2 we present the magnetization versus external applied magnetic field curves for several values of the angular width. These pictures present a typical profile of a magnetization curve of a mesoscopic superconductor. It presents a series of discontinuities, in which each jump signals the entrance of more vortices into the sample. Notice that the lower critical field does not vary with the shape of the circular sector. From this, the immediate conclusion is that it de- 

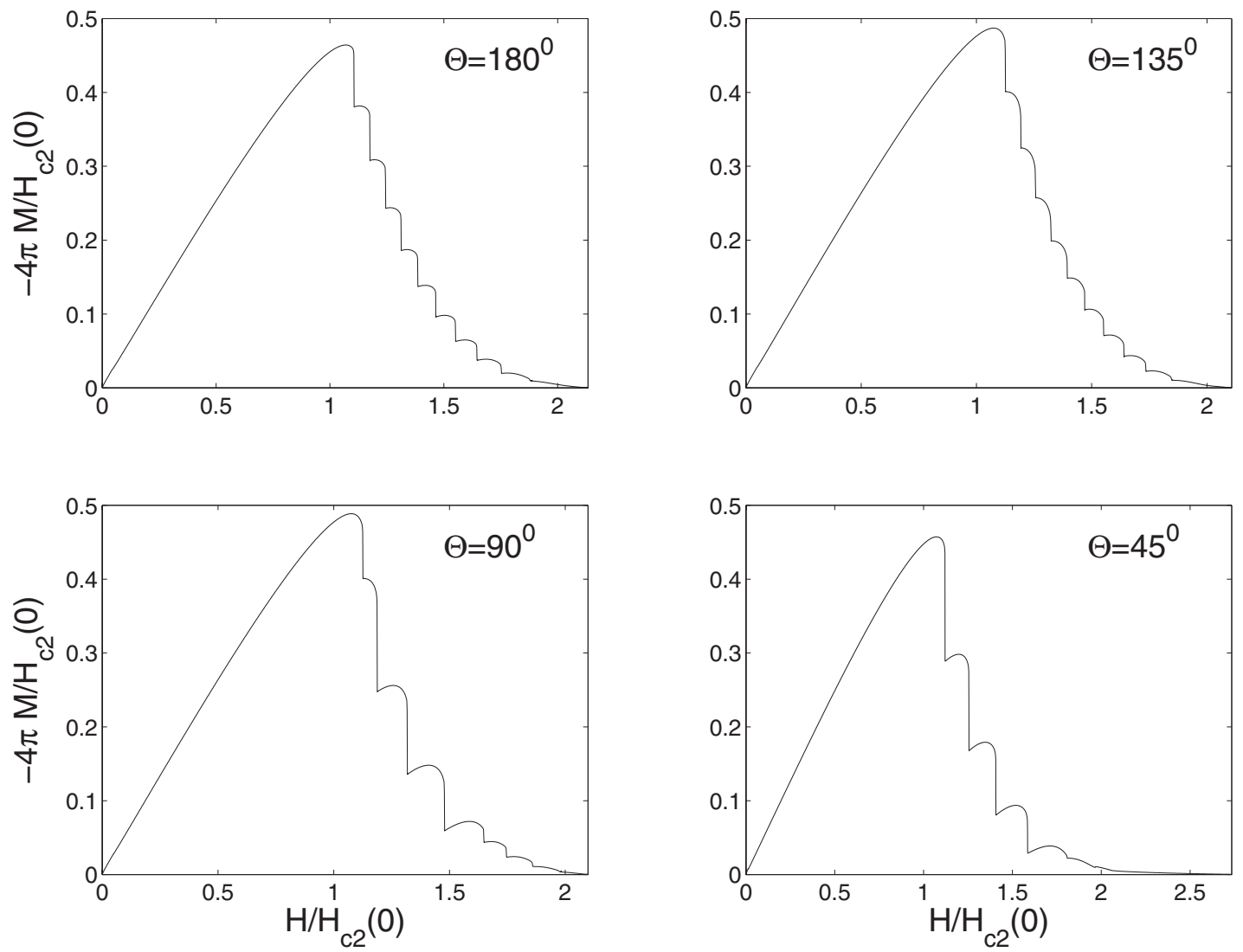

FIG. 2. The magnetization curve as a function of the external applied magnetic field for four values of the angular width of the circular sector. Each jump in the magnetization indicates a phase transition. The corresponding configuration for each phase is indicated in Table I.

pends only on the area but not on $\Theta$. In other words, our results indicate that, should we change the area to another value, the lower critical field would also change its value, but it would be the same for any value of $\Theta$. In fact, we determined the values of the lower critical field for a larger area which seems to corroborate this conclusion. In Fig. 3, we plotted $H_{c 1}(0)$ as a function of $\Theta$ for two different values of the area. As one would expect, the first nucleation field is slightly lower for the larger area. However, the fluctuations about the average value for a fixed area are very small in both cases.

Our result seems to be in good agreement with that found in Ref. 9 where numerical simulations were performed in three different geometries: disk, square, and triangle, having the same area, and using the same parameters as in the present contribution. The authors of this reference find that the magnitude of the lower critical field is the same for the disk and the square, and slightly larger for the triangle. Since we have the freedom to deform the circular sector, it should be expected a similar behavior for the lower critical field, as indeed it is indicated by Fig. 2. A possible explanation for the invariance of the lower critical field with the geometry is the following. The barrier for the nucleation of the first vortex inside the sample is related to the shielding currents. (For more details on this issue see Ref. 18.) Since the shielding currents depend on the area of the superconductor, one would expect the lower critical field to be the same for any geometry having the same area.
Another interesting feature present in the pictures of Fig. 2 is that the $\mathrm{SN}$ transition field $H_{c 3}(T)$ is approximately the same for all angles greater than $90^{\circ}$. However, for smaller values of $\Theta$, this critical field becomes significantly larger. Indeed, in Ref. $3 H_{c 3}(T)$ was calculated numerically for a

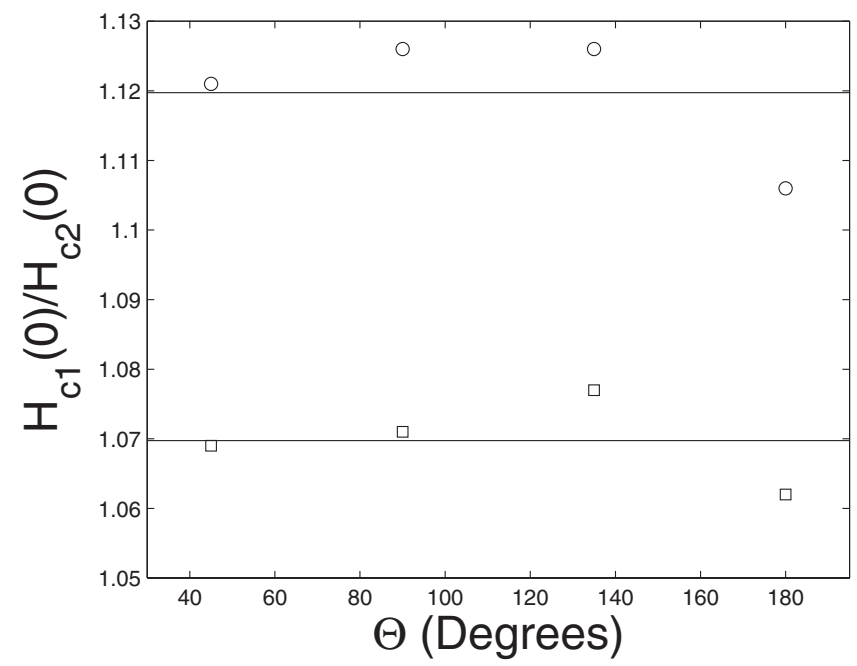

FIG. 3. The values of the lower critical field for two distinct values of the area: $\bigcirc, S=16 \pi$ and $\square, S=32 \pi$. The horizontal line indicates the average value of the nucleation field. The standard deviations from the averages are $9.5 \times 10^{-3}$ and $6.2 \times 10^{-3}$, respectively. 


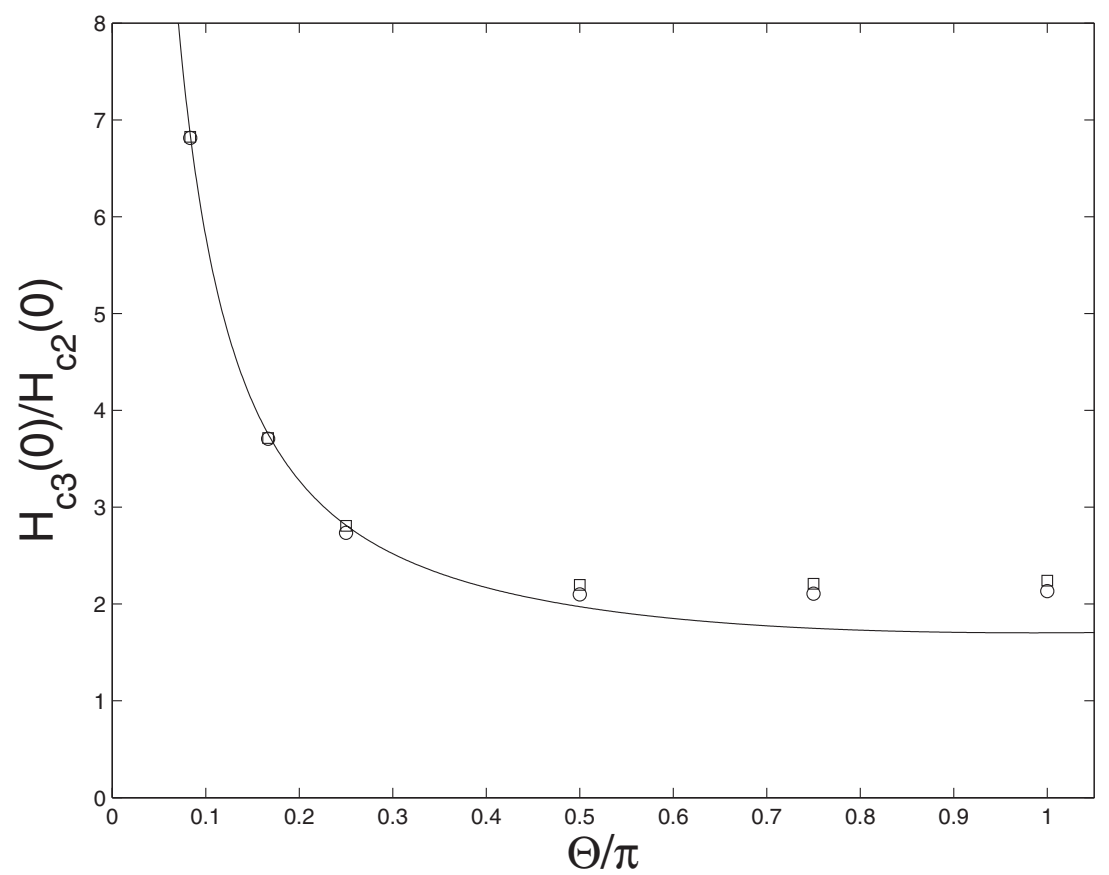

FIG. 4. The nucleation field as a function of the angular width of the circular sector. The solid line corresponds to Eq. (21) taken from Ref. 3 and the open circles and squares are the results found in the present simulation for two distinct areas: $\bigcirc, S=16 \pi$ and $\square$, $S=8 \pi$.

wedge. The area they used for the wedge is 2.24 larger than the one we used here. They found that their results fit quite well into the following expression:

$$
\frac{H_{c 3}(T)}{H_{c 2}(T)}=\frac{\sqrt{3}}{\Theta}\left(1+0.14804 \Theta^{2}+\frac{0.746 \Theta^{2}}{\Theta^{2}+1.8794}\right),
$$

where this expression is valid for any value of $T$, although our results are obtained for $T=0$. In fact, $H_{c 3}(T)$ is approximately the same for a disk and a square of equivalent area. ${ }^{9}$

In Fig. 4 we depict both the above expression and what we have found for the nucleation field $H_{c 3}(0)$ as a function of $\Theta / \pi$. As can be seen from that figure, for large angles, the nucleation field is larger in the geometry we consider. This suggests that, had we diminished the area of the circular sector, this difference for large angles would have increased. Nonetheless, for small angles all curves should collapse into a single curve, which corresponds to the asymptotic behavior $H_{c 3}(T) / H_{c 2}(T)=\sqrt{3} / \Theta$ determined in Ref. 3. This suggests that the superconductor behaves as a unidimensional system as $\Theta$ becomes small, no matter what the area is. Indeed, we determined $H_{c 3}(0)$ for a smaller area and found that the values of the nucleation field are slightly larger, but this difference tends to diminish for small $\Theta$ (see Fig. 4).

We also investigated the topology of the order parameter. Before going any further, let us establish the criterion we used to distinguish a single vortex from a giant vortex state. A giant vortex is nucleated as two or more vortices collapse into a single vortex in which all of them have, rigorously, a common core center. This was the criterion we used throughout this work which in a certain sense is more rigorous than others used in the literature (see, for instance, Ref. 9) where a giant vortex occurrence is accepted only when a local maximum of the order parameter between two minimums is lower than $0.5 \%$ of the maximum Cooper-pair density in the sample.
To describe an $N$ vortex state we used the following nomenclature. We denote by $N_{s} S$ a multiple vortex configuration formed by $N_{s}$ single vortices. A single giant vortex of vorticity $N_{g}$ is denoted by $1 G_{N_{g}}$. For example, the $4 S 1 G_{2}$ state is formed by four single vortices and a double quantized giant vortex.

In all geometries we have considered, usually it occurs for transitions either from $N$ to $N+1$ or from $N$ to $N+2$ vortices. In Figs. 5 and 6 we depict $|\psi|$ for $\Theta=180^{\circ}$ and $45^{\circ}$ respectively, and two stationary states with different values of $H$. We have chosen transitions where we could have the formation of a giant vortex. As can be seen in these figures, we have the transitions $9 S \rightarrow 4 S 1 G_{6} \quad\left(\Theta=180^{\circ}\right)$ and $6 S$ $\rightarrow 5 S 1 G_{3}\left(\Theta=45^{\circ}\right)$. On building these pictures we have used the highest resolution as possible in order to detect any individuality in the vortex configurations. Beyond some critical resolution, the pictures do not present a change.

On the other hand, if we look at the same pictures in a logarithm scale, we still see that the core centers of the vortices occupy different positions. So, within this criterion, we cannot affirm that a giant vortex has been nucleated. For higher vorticity, we have not observed any giant vortex either. A very different scenario takes place in disks, squares, and triangles even using the same parameters as in the present work. ${ }^{9,19}$ Maybe, for smaller areas, a giant vortex could be formed; we have not tested this possibility. All possible configurations are summarized in Table I up to $N=11$. Notice that the vortices are always symmetrically distributed along the mediatrix.

\section{SUMMARY}

In summary, an algorithm has been developed for solving the Ginzburg-Landau equations for circular geometries. This will probably make it much easier to extend the $\psi U$ method 

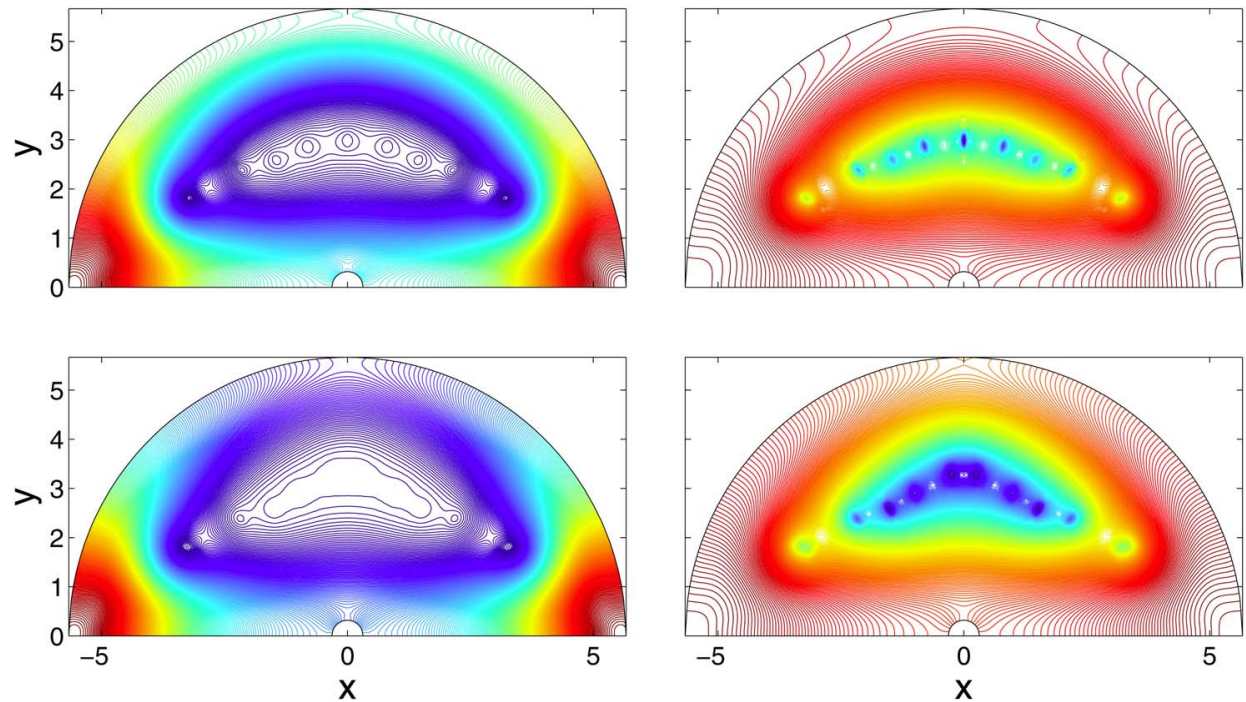

FIG. 5. (Color online) Two-dimensional density contour plots of $|\psi|$ for an angular width of $\Theta=180^{\circ}$ with $N=9$ and 10 vortices, from the top to the bottom (left column; the right column corresponds to the same pictures, but in logarithm scale). Both pictures correspond to the stationary states as the vortices enter the sample. Notice that once the equilibrium configuration is achieved, the vortices are symmetric with respect to the vertical axis. This feature is always present in the other geometries.

for other geometries in addition to the circular and rectangular ones. Furthermore, we have applied the algorithm to the circular sector and have found several configurations for the vortex state in this geometry. Also, the superconducting nucleation field has been evaluated. We have presented some evidence that, as we diminish the area of the supercondutor, the nucleation field increases. However, as the angular width goes to small values, this field exhibits a universal behavior regarded to the area.

\section{ACKNOWLEDGMENTS}

The authors thank the Brazilian Agencies FAPESP and
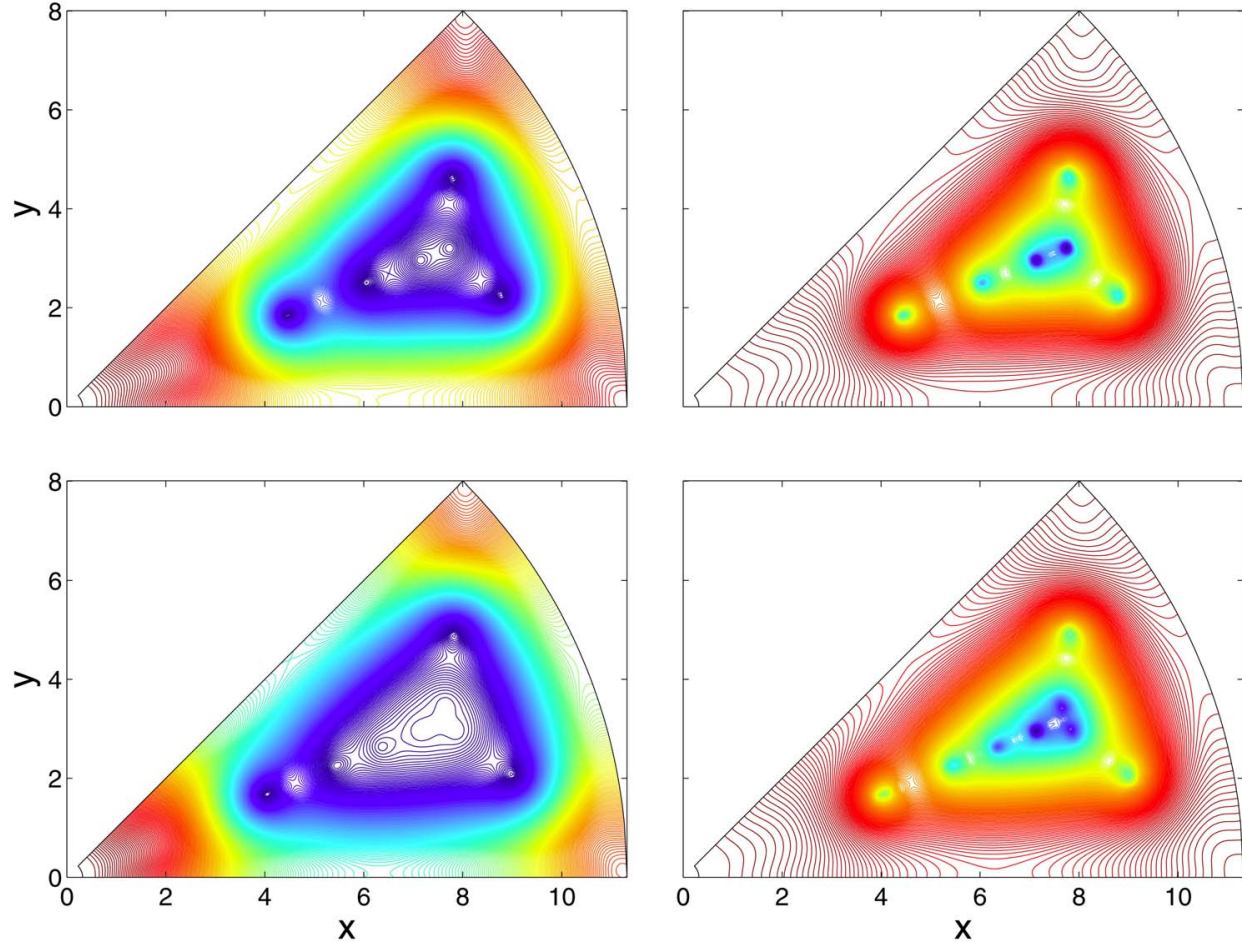

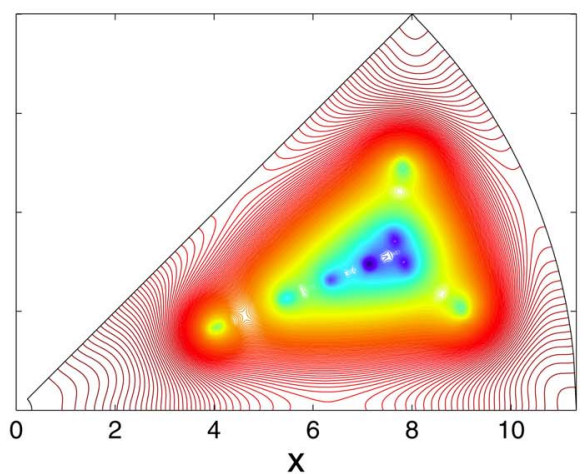

FIG. 6. (Color online) The same as Fig. 5 for $\Theta=45^{\circ}$ with $N=6$ and 8 . 
TABLE I. The sequence of vortex configurations for four different angles of the circular sector. The nomenclature used is explained in the text. The configurations in brackets correspond to what we obtain not using a logarithm scale.

\begin{tabular}{lrrrr}
\hline \hline$N$ & $180^{0}$ & $135^{0}$ & $90^{0}$ & $45^{0}$ \\
\hline 1 & $1 S$ & $1 S$ & $1 S$ & \\
2 & $2 S$ & $2 S$ & & $2 S$ \\
3 & $3 S$ & $3 S$ & $3 S$ & \\
4 & $4 S$ & $4 S$ & & \\
5 & $5 S$ & $5 S$ & $5 S$ & \\
6 & $6 S$ & $6 S$ & & \\
7 & $7 S$ & $7 S$ & $7 S\left(5 S 1 G_{2}\right)$ & \\
8 & $8 S$ & $8 S\left(6 S 1 G_{2}\right)$ & $8 S\left(5 S 1 G_{3}\right)$ & $8 S\left(5 S 1 G_{3}\right)$ \\
9 & $9 S$ & $9 S\left(4 S 1 G_{5}\right)$ & $9 S\left(5 S 1 G_{4}\right)$ & $9 S\left(4 S 1 G_{5}\right)$ \\
10 & $10 S\left(4 S 1 G_{6}\right)$ & $10 S\left(4 S 1 G_{6}\right)$ & $10 S\left(3 S 1 G_{7}\right)$ & $10 S\left(5 S 1 G_{5}\right)$ \\
11 & $11 S\left(5 S 1 G_{6}\right)$ & $11 S\left(2 S 1 G_{9}\right)$ & $11 S\left(3 S 1 G_{8}\right)$ & $11 S\left(4 S 1 G_{7}\right)$ \\
\hline \hline
\end{tabular}

CNPq for financial support and Clecio C. de Souza Silva for very useful discussions.

\section{APPENDIX A: DISCRETIZATION OF THE TDGL EQUATIONS}

The TDGL equations can be discretized by calculating them at the appropriate points according to the rules established in Fig. 1. This can be done by using the central difference approximation for the derivatives which is second order accurate in $\left(a_{\rho}, a_{\theta}\right)$. A tedious, however, straightforward calculation, leads us to the following discrete version of the TDGL equations of Eq. (8):

$$
\begin{gathered}
\frac{\partial \psi_{i, j}}{\partial t}=\mathcal{F}_{\psi, i, j}, \\
\beta \frac{\partial A_{\rho, i, j}}{\partial t}=(1-T) \operatorname{Im}\left[\frac{\bar{\psi}_{i, j} U_{\rho, i, j} \psi_{i+1, j}}{a_{\rho}}\right]-\kappa_{e f f}^{2}\left(\frac{h_{z, i, j}-h_{z, i, j-1}}{\rho_{i+1 / 2} a_{\theta}}\right), \\
\beta \frac{\partial A_{\theta, i, j}}{\partial t}=(1-T) \operatorname{Im}\left[\frac{\bar{\psi}_{i, j} U_{\theta, i, j} \psi_{i, j+1}}{\rho_{i} a_{\theta}}\right]+\kappa_{e f f}^{2}\left(\frac{h_{z, i, j}-h_{z, i-1, j}}{a_{\rho}}\right),
\end{gathered}
$$

where

$$
\begin{aligned}
\mathcal{F}_{\psi, i, j}= & \frac{\rho_{i+1 / 2}\left(U_{\rho, i, j} \psi_{i+1, j}-\psi_{i, j}\right)+\rho_{i-1 / 2}\left(\bar{U}_{\rho, i-1, j} \psi_{i-1, j}-\psi_{i, j}\right)}{\rho_{i} a_{\rho}^{2}} \\
& +\frac{U_{\theta, i, j} \psi_{i, j+1}-2 \psi_{i, j}+\bar{U}_{\theta, i, j-1} \psi_{i, j-1}}{\rho_{i}^{2} a_{\theta}^{2}}+(1-T) \psi_{i, j}(1 \\
& \left.-\left|\psi_{i, j}\right|^{2}\right) .
\end{aligned}
$$

From the numerical point of view, it is more convenient to evaluate the link variables rather than the vector potential. From Eqs. (12), we can easily verify that

$$
\frac{\partial A_{\rho, i, j}}{\partial t}=-\frac{\bar{U}_{\rho, i, j}}{i a_{\rho}} \frac{\partial U_{\rho, i, j}}{\partial t}, \quad \frac{\partial A_{\theta, i, j}}{\partial t}=-\frac{\bar{U}_{\theta, i, j}}{i \rho_{i} a_{\theta}} \frac{\partial U_{\theta, i, j}}{\partial t} .
$$

In addition, from Eq. (14), we can write, accurate to second order in $\left(a_{\rho}, a_{\theta}\right)$,

$$
h_{z, i, j}=\frac{\operatorname{Im}\left(1-L_{i, j}\right)}{a_{\rho} \rho_{i+1 / 2} a_{\theta}},
$$

where $L_{i, j}$ is given by Eq. (15). Upon introducing Eqs. (A3) and (A4) into the second and third equations of Eq. (A1) we obtain the following recurrence relations:

$$
\frac{\partial U_{\rho, i, j}}{\partial t}=-\frac{i}{\beta} U_{\rho, i, j} \mathcal{F}_{U_{\rho}, i, j}, \quad \frac{\partial U_{\theta, i, j}}{\partial t}=-\frac{i}{\beta} U_{\theta, i, j} \mathcal{F}_{U_{\theta}, i, j}
$$

where

$$
\begin{gathered}
\mathcal{F}_{U_{\rho}, i, j}=\operatorname{Im}\left[(1-T) \bar{\psi}_{i, j} U_{\rho, i, j} \psi_{i+1, j}+\kappa_{e f f}^{2}\left(\frac{L_{i, j}-L_{i, j-1}}{\rho_{i+1 / 2}^{2} a_{\theta}^{2}}\right)\right], \\
\mathcal{F}_{U_{\theta}, i, j}=\operatorname{Im}\left[(1-T) \bar{\psi}_{i, j} U_{\theta, i, j} \psi_{i, j+1}+\kappa_{e f f}^{2} \frac{\rho_{i}}{a_{\rho}^{2}}\left(\frac{L_{i-1, j}}{\rho_{i-1 / 2}}-\frac{L_{i, j}}{\rho_{i+1 / 2}}\right)\right] .
\end{gathered}
$$

Finally, on using the one-step forward-difference Euler scheme with time step $\Delta t$, we obtain the recurrence relations (16).

\section{APPENDIX B: PHYSICAL QUANTITIES}

The topology of the superconducting state is usually illustrated by $|\psi|^{2}$. This quantity can be determined from the outcome of the recurrence relations previously derived. Other important physical quantities used to describe the vortex state are the Gibbs free energy, the magnetization, and the vorticity. In what follows we will derive an expression for each of these physical quantities.

(1) The kinetic energy.

$$
\begin{aligned}
\mathcal{L}_{\mathrm{k}}= & (1-T) \iint_{\Omega_{\mathrm{SC}}}\left[\left|\frac{\partial\left(U_{\rho} \psi\right)}{\partial \rho}\right|^{2}+\left|\frac{1}{\rho^{2}} \frac{\partial\left(U_{\theta} \psi\right)}{\partial \theta}\right|^{2}\right] \rho d \rho d \theta=(1 \\
& -T) \sum_{i=2}^{N_{\rho}} \sum_{j=2}^{N_{\theta}} \int_{\theta_{j-1 / 2}}^{\theta_{j+1 / 2}} \int_{\rho_{i-1 / 2}}^{\rho_{i+1 / 2}}\left[\left|\frac{\partial\left(U_{\rho} \psi\right)}{\partial \rho}\right|^{2}\right. \\
& \left.+\left|\frac{1}{\rho^{2}} \frac{\partial\left(U_{\theta} \psi\right)}{\partial \theta}\right|^{2}\right] \rho d \rho d \theta=(1 \\
& -T) \sum_{i=2}^{N_{\rho}} \sum_{j=2}^{N_{\theta}}\left\{\frac { 1 } { 2 \rho _ { i } a _ { \rho } ^ { 2 } } \left[\rho_{i+1 / 2}\left|U_{\rho, i, j} \psi_{i+1, j}-\psi_{i, j}\right|^{2}\right.\right. \\
& \left.+\rho_{i-1 / 2}\left|U_{\rho, i-1, j} \psi_{i, j}-\psi_{i-1, j}\right|^{2}\right]+\frac{1}{2 \rho_{i}^{2} a_{\theta}^{2}}\left[\left|U_{\theta, i, j} \psi_{i, j+1}-\psi_{i, j}\right|^{2}\right. \\
& \left.\left.+\left|U_{\theta, i, j-1} \psi_{i, j}-\psi_{i, j-1}\right|^{2}\right]\right\} a_{\rho} a_{\theta} \rho_{i} .
\end{aligned}
$$

(2) The condensation energy. 


$$
\begin{aligned}
\mathcal{L}_{\mathrm{c}}= & (1-T)^{2} \iint_{\Omega_{\mathrm{SC}}}|\psi|^{2}\left(\frac{1}{2}|\psi|^{2}-1\right) \rho d \rho d \theta=(1 \\
& -T)^{2} \sum_{i=2}^{N_{\rho}} \sum_{j=2}^{N_{\theta}} \int_{\theta_{j-1 / 2}}^{\theta_{j+1 / 2}} \int_{\rho_{i-1 / 2}}^{\rho_{i+1 / 2}}|\psi|^{2}\left(\frac{1}{2}|\psi|^{2}-1\right) \rho d \rho d \theta=(1 \\
& -T)^{2} \sum_{i=2}^{N_{\rho}} \sum_{j=2}^{N_{\theta}}\left|\psi_{i, j}\right|^{2}\left(\frac{1}{2}\left|\psi_{i, j}\right|^{2}-1\right) a_{\rho} a_{\theta} \rho_{i} .
\end{aligned}
$$

(3) The field energy.

$$
\begin{aligned}
\mathcal{L}_{\mathrm{f}} & =\kappa_{\mathrm{eff}}^{2} \iint_{\Omega} h_{z}^{2} \rho d \rho d \theta=\kappa_{\mathrm{eff}}^{2} \sum_{i=1}^{N_{\rho}} \sum_{j=1}^{N_{\theta}} \int_{\theta_{j}}^{\theta_{j+1}} \int_{\rho_{i}}^{\rho_{i+1}} h_{z, i, j}^{2} \rho d \rho d \theta \\
& =\kappa_{\mathrm{eff}}^{2} \sum_{i=1}^{N_{\rho}} \sum_{j=1}^{N_{\theta}} \frac{\left[\operatorname{Im}\left(1-L_{i, j}\right)\right]^{2}}{a_{\rho}^{2} \rho_{i+1 / 2}^{2} a_{\theta}^{2}} a_{\rho} \rho_{i+1 / 2} a_{\theta} .
\end{aligned}
$$

The total Helmholtz energy is then given by $\mathcal{L}=\mathcal{L}_{\mathrm{k}}+\mathcal{L}_{\mathrm{c}}$ $+\mathcal{L}_{\mathrm{f}}$. The Gibbs free energy can be obtained by a simple modification in the field energy. Instead of $h_{z}^{2}$ we would have $\left(h_{z}-H\right)^{2}$, or $\left(h_{z, i, j}-H\right)^{2}$ in the discrete version. Notice that the discrete TDGL equations could also be derived through the following equations:

$$
(1-T) \frac{\partial \psi_{i, j}}{\partial t}=-\frac{1}{\mathcal{A}_{i}} \frac{\partial \mathcal{L}}{\partial \bar{\psi}_{i, j}}
$$

$$
\beta \frac{\partial A_{\alpha, i, j}}{\partial t}=-\frac{1}{2 \mathcal{A}_{\alpha, i}} \frac{\partial \mathcal{L}}{\partial A_{\alpha, i, j}},
$$

where $\mathcal{A}_{i}=a_{\rho} \rho_{i} a_{\theta}, \mathcal{A}_{\rho, i}=a_{\rho} \rho_{i+1 / 2} a_{\theta}$, and $\mathcal{A}_{\theta, i}=a_{\rho} \rho_{i} a_{\theta}$, which are the areas surrounded by the vertex and link points, respectively. In order to derive the discrete TDGL equations by this means, it is essential to use the following relations:

$$
\frac{\partial U_{\rho, i, j}}{\partial A_{\rho, i, j}}=-i a_{\rho} U_{\rho, i, j}, \quad \frac{\partial U_{\theta, i, j}}{\partial A_{\theta, i, j}}=-i \rho_{i} a_{\theta} U_{\theta, i, j},
$$

which can be easily shown from Eqs. (12).

The magnetization is $4 \pi M=B-H$, where $B$ is the magnetic induction which is given by the spatial average of the local magnetic field. We have

$$
4 \pi M=\frac{1}{\mathcal{A}} \sum_{i=1}^{N_{\rho}} \sum_{j=1}^{N_{\theta}} h_{z, i, j} \mathcal{A}_{\rho, i}-H
$$

where $\mathcal{A}$ is total area of the circular sector.

The vorticity can be determined by integrating the phase $\varphi$ in each unit cell of the mesh. We have

$$
N_{i, j}=\frac{1}{2 \pi} \oint_{\mathcal{C}_{i, j}} \nabla \boldsymbol{\varphi} \cdot d \mathbf{r}, \quad N=\sum_{i=1}^{N_{\rho}} \sum_{j=1}^{N_{\theta}} N_{i, j},
$$

where $\mathcal{C}_{i, j}$ is a closed path with the lower left and upper right corner at $(i, j)$ and $(i+1, j+1)$, respectively. In all of our numerical simulations described previously, we calculated $N$ in order to make sure that the number of vortices agrees with what we see on the topological map of the order parameter.
${ }^{1}$ G. R. Berdiyorov, B. J. Baelus, M. V. Milosević, and F. M. Peeters, Phys. Rev. B 68, 174521 (2003).

${ }^{2}$ T. Puig, E. Rosseel, M. Baert, M. J. VanBael, V. V. Moshchalkov, and Y. Bruynseraede, Appl. Phys. Lett. 70, 3155 (1997); T. Puig, E. Rosseel, L. Van Look, M. J. Van Bael, V. V. Moshchalkov, Y. Bruynseraede, and R. Jonckheere, Phys. Rev. B 58, 5744 (1998); V. Bruyndoncx, J. G. Rodrigo, T. Puig, L. Van Look, V. V. Moshchalkov, and R. Jonckheere, ibid. 60, 4285 (1999); see also V. V. Moshchalkov et al., in Connectivity and Superconductivity, edited by J. Berger and J. Rubinstein (Springer, Heidelberg, 2000).

${ }^{3}$ V. A. Schweigert and F. M. Peeters, Phys. Rev. B 60, 3084 (1999); A. P. van Gelder, Phys. Rev. Lett. 20, 1435 (1968).

${ }^{4}$ A. Kanda, B. J. Baelus, F. M. Peeters, K. Kadowaki, and Y. Ootuka, Phys. Rev. Lett. 93, 257002 (2004).

${ }^{5}$ T. Nishio, S. Okayasu, J. Suzuki, and K. Kadowaki, Physica C 412-414, 379 (2004).

${ }^{6}$ S. Okayasu, T. Nishio, Y. Hata, J. Suzuki, I. Kakeya, K. Kadowaki, and V. V. Moshchalkov, IEEE Trans. Appl. Supercond. 15, 696 (2005).

${ }^{7}$ I. V. Grigorieva, W. Escoffier, V. R. Misko, B. J. Baelus, F. M. Peeters, L. Ya. Vinnikov, and S. V. Dubonos, Phys. Rev. Lett.
99, 147003 (2007).

${ }^{8}$ E. Sardella, A. L. Malvezzi, P. N. Lisboa-Filho, and W. A. Ortiz, Phys. Rev. B 74, 014512 (2006).

${ }^{9}$ B. J. Baelus and F. M. Peeters, Phys. Rev. B 65, 104515 (2002).

${ }^{10}$ A. Schmid, Phsys. Kondens. Materie 5, 302 (1966).

${ }^{11}$ W. D. Gropp, H. G. Kaper, G. K. Leaf, D. M. Levine, M. Palumbo, and V. M. Vinokur, J. Comput. Phys. 123, 254 (1996).

${ }^{12}$ G. C. Buscaglia, C. Bolech, and A. López, Connectivity and Superconductivity, edited by J. Berger and J. Rubinstein (Springer, New York, 2000).

${ }^{13}$ V. A. Schweigert and F. M. Peeters, Phys. Rev. B 57, 13817 (1998).

${ }^{14}$ J. Pearl, Appl. Phys. Lett. 5, 65 (1964).

${ }^{15}$ G. R. Berdiyorov, M. V. Milosevic, and F. M. Peeters, Physica C 437-438, 25 (2006).

${ }^{16}$ Qiang Du, J. Comput. Phys. 67, 965 (1998).

${ }^{17}$ J. Berger, J. Comput. Phys. 46, 095106 (2005).

${ }^{18}$ A. K. Geim, S. V. Dubonos, I. V. Grigorieva, K. S. Novoselov, F. M. Peeters, and V. A. Schweigert, Nature (London) 407, 55 (2000).

${ }^{19}$ V. A. Schweigert, F. M. Peeters, and P. S. Deo, Phys. Rev. Lett. 81, 2783 (1998). 